\title{
DESIGN AND ANALYSIS OF MIMO SYSTEM FOR UWB COMMUNICATION
}

\author{
Mihir N. Mohanty ${ }^{1}$, Monalisa Bhol ${ }^{2}$, Laxmi Prasad Mishra ${ }^{3}$, Sanjat Kumar \\ Mishra $^{4}$
}

1ITER, Siksha 'O' Anusandhan University, Bhubaneswar, Odisha, 751030, India

Seemanta Engineering College, Jharpokharia, Mayurbhanj, Odisha, India

\begin{abstract}
Multiple transmit and receive antennas are used MIMO system. The system creates parallel MIMO subchannels to transmit independent streams of data under the appropriate channel conditions. Similarly, Ultrawideband (UWB) communication has attracted great interest for various applications in recent days. Spatially multiplexed (SM) multiple-input multiple-output (MIMO) systems gains the spectral efficiency as well as high data rates without consuming additional power, bandwidth or time slots. In this paper, we extend the concept of MIMO to UWB systems. The correlated channel for such purpose is considered and the performance has been analyzed for spatial multiplexing SM-UWB-MIMO system which is required for estimation. The system performance substantially degrades in the presence of high values of spatial correlation. To avoid the degradation of such system, it has been designed for virtual UWB-MIMO Time Reversal (TR) system, so that it is not affected by the transmit correlation. Another novel method to reduce the effect of correlation has been chosen by taking the Eigen value of the channel matrix for the computation of the system performance. The result shows its performance.
\end{abstract}

\section{KEYWORDS}

MIMO, UWB, BER, Channel Capacity, Spatial Correlation, TR, Eigen Value.

\section{INTRODUCTION}

Wireless propagation channels have been investigated for more than two decades and a large number of channel models are designed by many researchers. Ultrawideband (UWB) communication system has become most promising for high data rate as well as short-range communication systems. Therefore, it has attracted great interests from both academic and industrial aspects recently. Because of the restrictions on the transmit power, UWB communications are best suited for short-range communications [1].

Increasing demand for higher wireless system capacity has catalyzed several transmission techniques, among which multiple-input/multiple-output (MIMO) technology is popular one. Extending MIMO technology to the UWB regime, a large gain in the channel capacity, robustness and coverage radius is noticed in UWB indoor communications systems [2]. These systems are equipped with multiple antennas, at both the transmitter and receiver in order to improve communication performance, in contrast to conventional communication systems with only one antenna on the transmitter and one antenna on the receiver. 
MIMO technologies overcome the deficiencies of the traditional methods through the use of spatial diversity. Data can be transmitted over $\mathrm{M}$ transmit antennas to $\mathrm{N}$ receive antennas supported by the receiver terminal. Such systems are used in wireless communication for enhancement of capacity and bit error rate (BER). It offers significant increases in data throughput and link range without additional bandwidth or transmit power. These characteristics are essential for the coming generation of Telecommunications systems. Rayleigh fading has been considered as the propagation channel for verification. Diversity gain and spatial multiplexing (SM) are the two main advantages of MIMO systems that are used to study the effect of increase in bit rate with increasing the number of transmitter and receiver antennas. In MIMO system, we primarily need to take into account the spatial correlation. The effect of spatial correlation has to be minimized to obtain better system performance. In [3], the time-reversed channel impulse response (CIR) is implemented as a filter at the transmitter side. It is well known that the MIMOTR-UWB system can achieve transmit diversity, but it suffers from both transmit and receive antenna correlations. The single-input multiple-output TR-UWB (SIMO-TR-UWB) or virtual MIMO-TR-UWB does not face the transmit antenna correlation because it has only one transmit antenna.

\section{RELATED LITERATURE}

An overview of reported measurements and modelling of the UWB indoor wireless channel is presented in [4]. Different UWB channel sounding techniques are discussed and approaches for the modelling of the UWB channel are reviewed.

A considerable work has been performed in [5-7] to characterize communication channels for general wireless applications. As MIMO systems operate at an unprecedented level of complexity to exploit the channel space-time resources, a new level of understanding of the channel spacetime characteristics is required to assess the potential performance of practical multi-antenna links.

Empirical investigation of spatial correlation in UWB indoor channels has been presented in [8]. It was observed that the coherence distance falls with channel bandwidth in end-fire arrays but not in broadside arrays. The complex correlation decays less rapidly with distance in broadside arrays than in end fire arrays, especially under line-of-sight. Strong dependence of spatial correlation and coherence distance on the channel centre frequency was observed.

Spatial multiplexing single-input-multiple-output (SM-SIMO) UWB communication system using the TR technique has been proposed. The system with only one transmit antenna, using a spatial multiplexing scheme, can transmit several independent data streams to achieve a very high data rate. TR can mitigate not only the ISI but the MSI caused by multiplexing several data streams simultaneously as well [9]. Antenna selection scheme for MIMO UWB communication system with TR is investigated in [10].

Time reversal technique has advantage in highly scattering environments to achieve signal focusing through transmitter-side processing that enables the use of simple receivers. The authors have also demonstrated UWB time reversal system architecture taking into account some practical constraints [11]. 


\section{Methodology}

A tractable correlated MIMO UWB channel model is essential when developing multiple-antenna UWB systems in order to accurately predict their performance. The system is designed based on Alamouti code. Figure 1 show for a 2 X2 antenna system. In Alamouti encoding scheme, during any given transmission period two signals are transmitted simultaneously from two transmit antennas.

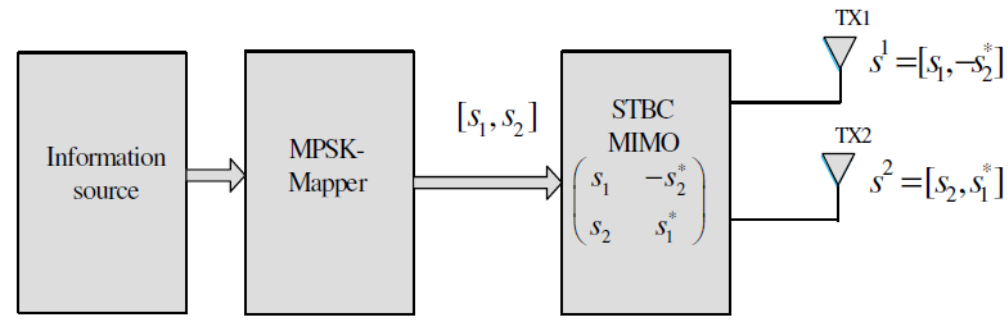

Fig. 1. Encoder for Alamouti schemes

At time $\mathrm{t}$, antenna 1 transmits $\mathrm{s} 1$, and simultaneously, antenna 2 transmits $\mathrm{s} 0$. At time $\mathrm{t}+\mathrm{T}$, where $\mathrm{T}$ is the symbol duration, signal transmission is switched, with $-\mathrm{s} 0 *$ transmitted by antenna 1 and $\mathrm{s} 1 *$ simultaneously, transmitted by antenna 2 . We present the measurements of a MIMO system under line-of-sight conditions.

\subsection{Spatial Correlation}

Though the space-time focusing feature is one of the benefits, spatial multiplexing has a major role in MIMO systems. Without the expansion of bandwidth, high data rate can be achieved by using spatial multiplexing scheme with multiple transmit and receive antennas. The spatial correlation in the multipath channel is a critical factor in the performance of a MIMO system and is evaluated. It is mainly caused by inadequate antenna spacing in both transmitting and receiving side. It causes correlation between the received signals, which degrades the signal quality, capacity and bit error rate (BER) performance. Capacity increases and BER performance also increases as signal correlation decreases. The fading correlation between the array elements should be sufficiently low for a MIMO system to offer any performance enhancement.

The MIMO channel matrix is assumed to be independent of each other. Other assumptions that can be made for such model analysis is as follows:

The correlation among the receive antennas is independent of the correlation between the transmit antennas.

The effect of antenna coupling is neglected, and we focus only on the spatial correlation.

The transmit and receive correlation matrices are fixed.

The correlation has been included to the MIMO UWB channel model by introducing fixed transmit and receive correlation matrices following the well-known Kronecker model [12-14],

$$
H=R_{r x}^{1 / 2} H_{W} R_{t x}^{1 / 2}
$$

where, $\quad H_{w}=$ channel matrix of independent channel realization.

$R_{t x}^{1 / 2}=$ transmit correlation matrix with dimension $M \times M$.

$R_{r x}^{1 / 2}=$ receive correlation matrix with dimension $N$ x $N$. 


$$
H=\text { correlated channel }
$$

\subsection{Methods for Computing Spatial Correlation}

1. Gathering a large amount of data in the target propagation environment. For that it becomes necessary to estimate a large number of correlation coefficients in an M x N MIMO system. Suppose, there are MN spatial sub-channels, and correlating each pair of them would give rise to $(M N)^{2}$ correlation values. A disadvantage of this approach, beside the fact that it may be very time-consuming, is that it may be necessary to estimate a large number of correlation coefficients.

2. Using fixed correlation matrices as:

i. Transmit correlation matrix

ii. Receive correlation matrix

The transmit correlation matrix is given as:

$$
R_{t x}=\left[\begin{array}{cccc}
1 & \rho_{T x} & \rho_{T x}^{2} \cdots & \rho_{T x}^{M-1} \\
\rho_{T x} & 1 & \rho_{I x} \cdots & \rho_{T x}^{M-2} \\
\vdots & \vdots & \ddots & \vdots \\
\rho_{T x}^{M-1} & \rho_{T x}^{M-2} & \rho_{T x}^{M-3} \cdots & 1
\end{array}\right]
$$

Similarly, receive correlation matrix is given as:

$$
R_{r x}=\left[\begin{array}{cccc}
1 & \rho_{R x} & \rho_{R x}^{2} \cdots & \rho_{R x}^{N-1} \\
\rho_{R x} & 1 & \rho_{R x} \cdots & \rho_{R x}^{N-2} \\
\vdots & \vdots & \ddots & \vdots \\
\rho_{R x}^{N-1} & \rho_{R x}^{N-2} & \rho_{R x}^{N-3} \cdots & 1
\end{array}\right]
$$

The advantage of the fixed correlation model is its simplicity and its immediate application to the existing IEEE 802.15.3a standard.

The fixed correlation matrices $R_{t x}$ and $R_{r x}$ appropriate for a particular environment can be determined by selecting the numerical values of $\rho_{T x}$ (transmit correlation coefficient) and $\rho_{R x}$ (receive correlation coefficient), such that a close match is obtained to the BER results achieved when conducting the system simulation using the measured indoor channel. The correlation coefficient values ranges from 0 to 1 . We present BER results for various MIMO UWB systems for various values of the channel correlation coefficient.

\subsection{Proposed Method for Reduction of Correlation Effect}

\section{Virtual MIMO-UWB-Time Reversal Technique System}

The time reversal (TR) technique, which is originated from under-water acoustics and ultrasonic, now has been used in many applications such as localization, imaging and green wireless communications. TR also has shown its potential in dealing with the ISI problems in UWB. In a TR system, the time-reversed channel response (CIR) is implemented as a filter at the transmitter side [15].

With the help of the TR pre-filter, the system with only one transmit antenna can deliver several independent data streams at the same time. We have taken the spatial correlation into account in, 
where a constant spatial correlation model for MIMO UWB has been applied. The performance of system over correlated line of- sight (LOS) channels is investigated with the same correlation model. This scenario is referred to as channel model1 (CM1) in the IEEE 802.15.3a standard. The BER results on the adopted correlated channel model with an appropriate value of correlation coefficient are shown closely matching with those on the measured indoor channel.

It is well known that the MIMO-TR-UWB system can achieve transmit diversity, but it suffers from penalty caused by both transmit and receive antenna correlations. Meanwhile, the singleinput multiple-output TR-UWB (SIMO-TR-UWB) or virtual MIMO-TR-UWB does not face the transmit antenna correlation because it has only one transmit antenna. It is shown the virtual MIMO outperforms the true MIMO system in terms of the BER performance. The channel impulse response (CIR) between the transmit antenna $j$ and the receive antenna $i$ is, [15],

$$
h_{i, j}(t)=\alpha^{i, j} \delta\left(t-\tau^{i, j}\right)
$$

where, $\alpha$ is the amplitude

$\tau$ is the delay and the value is considered for it as IEEE 802.15.3a standard from the Table-1

$$
H_{i . j}(t)=\left(\begin{array}{cccc}
h_{1,1}(t) & h_{1,2}(t) & \cdots & h_{1, M}(t) \\
h_{2,1}(t) & h_{2,2}(t) & \cdots & h_{2, M}(t) \\
\vdots & \vdots & \ddots & \vdots \\
h_{N, 1}(t) & h_{N, 2}(t) & \cdots & h_{N, M}(t)
\end{array}\right)
$$

The TR pre-filter matrix of the MIMO system is given by:

where,

$$
\overline{H_{i, j}}(t)=\left(\begin{array}{cccc}
\overline{h_{1,1}}(-t) & \overline{h_{2,1}}(-t) & \cdots & \overline{h_{N, 1}}(-t) \\
h_{1,2}(-t) & \overline{h_{2,2}}(-t) & \cdots & \overline{h_{N, 2}}(-t) \\
\vdots & \vdots & \ddots & \vdots \\
\overline{h_{1, M}}(-t) & \overline{h_{2, M}}(-t) & \cdots & \overline{h_{N, M}}(-t)
\end{array}\right)
$$

$$
\bar{h}(t)=h(t) \otimes h(-t)
$$

The matrix of the equivalent channel is a square matrix with the entries in the main diagonal being the summation of the autocorrelation of the original CIRs and other entries being the summation of the cross-correlation of the original CIRs between the transmit and receive antennas.

The TR matrix $\overline{H_{i . j}}(t)$ is used instead of $H_{W}(t)$ in equation (1), to calculate the BER performance of the MIMO UWB system. The following parameters have been considered for evaluation of UWB channel model. It has been tested according to the IEEE standard 802.15.3a UWB model.

Table 1. Channel model Parameter of IEEE 802.15.3a standard.

\begin{tabular}{|l|l|}
\hline Parameters & Specific Values considered \\
\hline Channel Model & CM1 for Line of Sight communication \\
\hline Frequency & $3 \mathrm{GHz}$ \\
\hline Channel & Rayleigh fading channel \\
\hline Modulation $\tau$ & QPSK 5.05 ns \\
\hline
\end{tabular}


It is shown the virtual MIMO outperforms the true MIMO system in term of the BER performance. Another method to reduce the effect of correlation has been chosen by taking the Eigen value of the channel matrix for the computation of the system performance.

\section{Eigen Values of the Correlation Matrix}

The sub-channel correlation, power gains of supported eigen modes, and branch power ratios are analyzed. The mutual information capacity is found to scale almost linearly with the MIMO array size, with very low variance. Eigen value of the correlation matrix is considered, that can be expressed as the relation:

$$
\operatorname{det}(\lambda I-A)=0
$$

where, $\lambda=$ the eigen value of $A$ and $A=$ square matrix

\section{RESULTS AND DISCUSSION}

In Fig. 2, shows the performance of channel capacity in the wireless channel. The result is measured according to the various SNR values. The capacity of the MIMO channel has been simulated for number of transmitter and receiver antennas, such as $2 \times 2,3 \times 3,4 \times 4$, and $8 \times 8$ MIMO systems. It is observed that capacity gradually increases with the number of antennas. Fig. 3 , i.e., BER versus SNR, with different number of transmitting and receiving antennas $(2 \times 2,3 \times$ $3,4 \times 4$, and $8 \times 8$ ). It is seen that the BER performance increases gradually with the increase in the number of antennas.

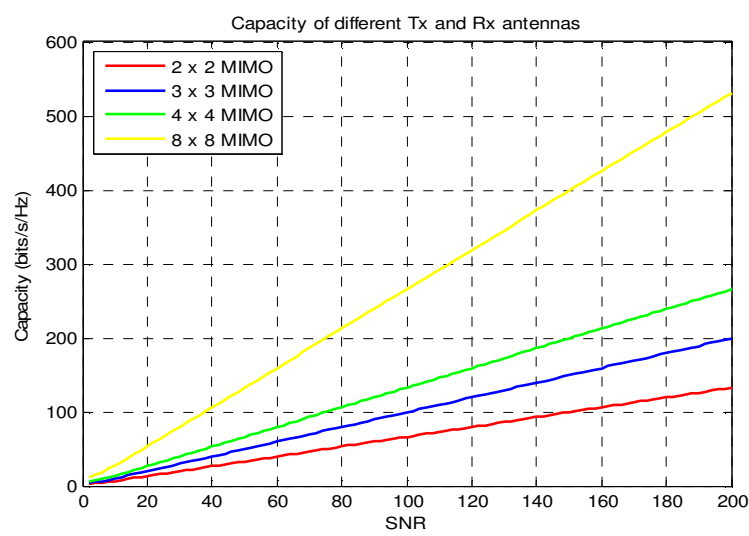

Fig. 2. Performance of capacity with respect to SNR 
International Journal of Wireless \& Mobile Networks (IJWMN) Vol. 6, No. 2, April 2014

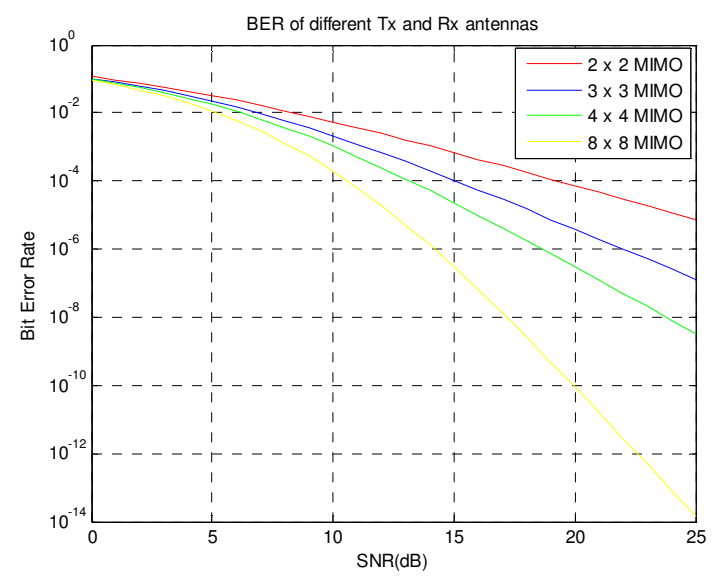

Fig. 3 Performance of BER

Fig. 4 shows the capacity results for $M=N=2$, that is $(2 \times 2)$ for the systems operating in the CM1 with correlation coefficients $\rho_{T x}$ and $\rho_{R x}$ to be $0,0.3$ and 0.9 in the measured UWB LOS channel. The capacity for different correlation factors is tested and it has been found that the capacity decreases with increase in the correlation factors and also decreases with increase in the number of correlated antenna elements. BER results for $M=N=2$, that is (2 $\mathrm{x} 2$ ) for the systems operating in the CM1 with correlation coefficients $\rho_{T x}$ and $\rho_{R x}$ to be $0,0.3$ and 0.9 and without applying time reversal in the measured UWB-LOS channel is shown in Fig. 5.

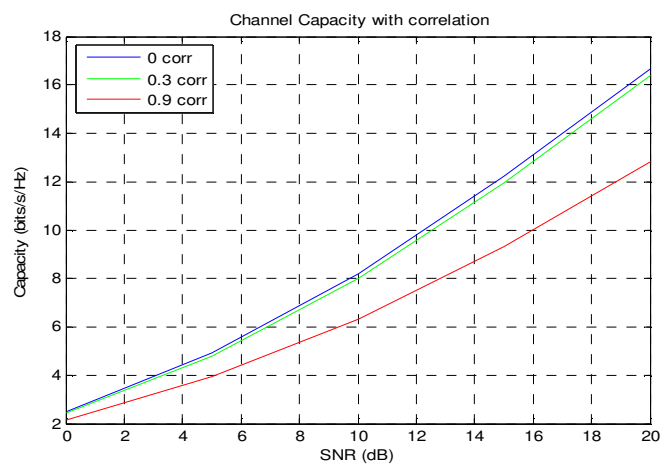

Fig.4. Capacity performance with correlation in $2 \times 2$ systems

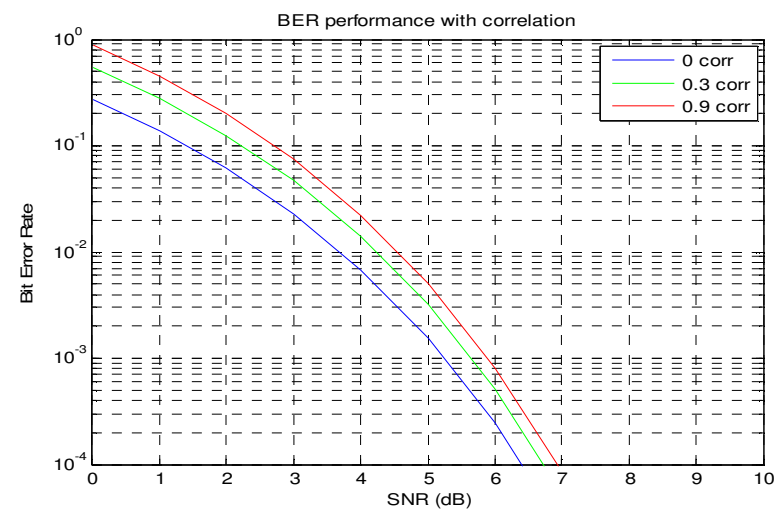

Fig. 5. BER performance with correlation 
It has been observed that the BER performance decreases with increase in the value of the correlation coefficients.

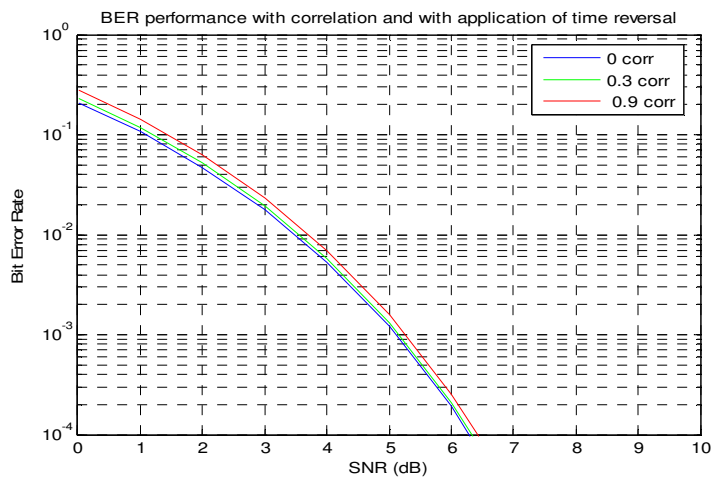

Fig. 6. BER performance with correlation and time reversal technique

The BER results for $M=1, N=2$, that is $(1 \times 2)$ for the systems operating in the CM1 with correlation coefficients $\rho_{T x}$ and $\rho_{R x}$ to be $0,0.3$ and 0.9 is shown in Fig. 6, by applying virtual MIMO time reversal in the measured UWB -LOS channel. Also it has been shown that the BER performance is better than the one without time reversal.

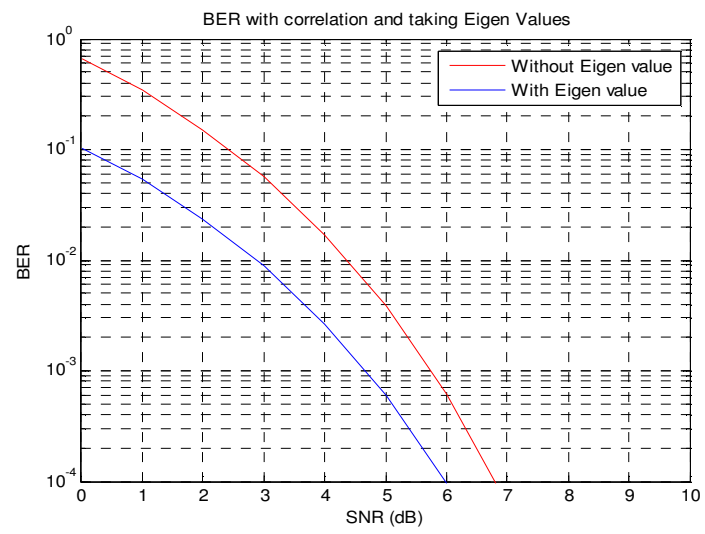

Fig. 7. BER with Eigen values

In Fig. 7, BER performance of $2 \times 2$ MIMO-UWB systems in the LOS indoor CM1 with correlation coefficients $\rho_{T x}=\rho_{R x}=0.4$ is shown. The comparison of the BER shows its efficacy. It is considered with correlation and the Eigen value of the correlation matrix in shown. Here, it has been observed that, the BER performance is even better for eigen values of the correlation matrix if considered.

\section{CONCLUSION}

Multiple antenna communications technologies offer significant advantages over single antenna systems. We have proposed different methods to reduce the impact of correlation in the MIMOUWB channel and presented a comprehensive comparative analysis with a distance independent spatial correlation model. Comparison between the BER performance of a system with correlation using virtual MIMO time reversal technique and without using time reversal technique is evaluated. BER performance of the system with eigen values and without eigen values of the 
correlation matrix respectively are also evaluated. Capacity result for 2 x 2 systems with different correlation coefficient values are shown as the proof. These advantages include extended range, improved reliability in fading environments and higher data throughputs.

\section{REFERENCES}

[1] Andreas F. Molisch., Jeffrey R. Foerster.: Channel Models For Ultrawideband Personal Area Networks,Vol. 10. IEEE Wireless Communications (2003) 14-21.

[2] Wasim Q. Malik., David J. Edwards.: Measured MIMO Capacity and Diversity Gain With Spatial and Polar Arrays in Ultrawideband Channels,Vol. 55. IEEE Transactions On Communications (2007) 2361-2370.

[3] T. K. Nguyen., H. Nguyen, F. Zheng., T. Kaiser.: Spatial correlation in SM-MIMO-UWB systems using a pre-equalizer and pre-Rake filter, Proceedings of the IEEE International Conference on Ultra-Wideband (ICUWB) (2010) 540-543.

[4] Zoubir Irahhauten., Homayoun Nikookar., Gerard J. M. Janssen.: An Overview Of Ultra Wide Band Indoor Channel Measurements And Modeling, Vol. 14. IEEE Microwave And Wireless Components Letters (2004) 386-38.

[5] Mihir Narayan Mohanty., Sikha Mishra.: Design of MCM based Wireless System using Wavelet Packet Network \& its PAPR Analysis, IEEE Conference, ICCPCT-2013 (2013) 821-824.

[6] Mihir Narayan Mohanty., Laxmi Prasad Mishra., Saumendra Kumar Mohanty.: Design of MIMO Space-Time Code for High Data Rate Wireless Communication, Vol. 3, No. 2. IJCSE, (2011) 693696.

[7] Michael A. Jensen., Jon W. Wallace.: MIMO Wireless Channel Modeling and Experimental Characterization, Space-Time Processing for MIMO Communications, John Wiley \& Sons, Ltd (2005) 1-39.

[8] Wasim Q. Malik.: Spatial Correlation in Ultrawideband Channels, Vol. 7. IEEE Transactions On Wireless Communications (2008) 604-610.

[9] Hieu Nguyen., Zhao Zhao, Feng Zheng., Thomas Kaiser.: Preequalizer Design for Spatial Multiplexing SIMO-UWB TR Systems,Vol.59. IEEE Transactions On Vehicular Technology (2010) 3798-3805.

[10] Hieu Nguyen., Feng Zheng., Thomas Kaiser.: Antenna Selection for Time Reversal MIMO UWB Systems, IEEE Transactions on Signal Processing (2009) 1-5.

[11] Nan Guo., Brian M. Sadler., Robert C. Qiu.: Reduced-Complexity UWB Time-Reversal Techniques and Experimental Results,Vol. 6. IEEE Transactions on Wireless Communications (2007) 1-6.

[12] T. Kaiser., F. Zheng., E. Dimitrov.: An Overview of Ultrawide-band Systems with MIMO,Vol. 97. Proceedings of the IEEE (2009) 285-312.

[13] A. Paulraj., R. Nabar., D. Gore.: Introduction to Space-Time Wireless Communications, Cambridge University Press (2003).

[14] S. L. Loyka.: Channel Capacity of MIMO Architecture Using the Exponential Correlation Matrix, Vol. 5. IEEE Communications Letters (2001) 369-371.

[15] Hieu Nguyen., Van Duc Nguyen., Trung Kien Nguyen., Kiattisak Maichalernnukul., Feng Zheng., Thomas Kaiser.: On the Performance of the Time Reversal SM-MIMO-UWB System on Correlated Channels, Hindawi Publishing Corporation International Journal of Antennas and Propagation (2012) 1-8. 